\title{
Adaptive Sliding Mode Controller Design For Attitude Small UAV
}

\author{
Samaneh Amini, Ali Akbar Akbari \\ Department of Mechanical Engineering, Ferdowsi University of Mashhad, Iran
}

\begin{tabular}{l} 
Article Info \\
\hline Article history: \\
Received Apr 6, 2015 \\
Revised Aug 18, 2015 \\
Accepted Aug 26, 2015 \\
\hline
\end{tabular}

\section{Keyword:}

Adaptation Law

Sliding Mode

Small Fixed Wing UAV

\begin{abstract}
The dynamic of Unmanned Aerial Vehicle (UAV) is nonlinear, strongly coupled, multi-input multi-output (MIMO), and subject to uncertainties and external disturbances. In this paper, an adaptive sliding mode controller (ASMC) is integrated to design the attitude control system for an inner loop fixed wing UAV. In the proposed scheme, sliding mode control law parameters due to uncertainty are assumed to be unknown and are estimated via adaptation laws. The synthesis of the adaptation laws is based on the positivity and Lyapunov design principle. Navigation outer loop parameters are regulated via PID controllers. Simulation results indicate that the proposed controller design can stabilize the nonlinear system, and it is robust to parametric model uncertainties and external disturbance.
\end{abstract}

Copyright $(0) 2015$ Institute of Advanced Engineering and Science. All rights reserved.

Corresponding Author:

Samaneh Amini, Department of Mechanical Engineering,

Ferdowsi University of Mashhad, Iran,

Email: amini.samane@ymail.com

\section{INTRODUCTION}

An unmanned aerial vehicle (UAV) is a powered, aerial vehicle that does not carry a human operator, uses aerodynamic forces to provide vehicle lift, can fly autonomously or piloted remotely, and can carry payloads [1].

In recent years, micro and small UAVs have attracted many researchers and developers around the world since they have the potential to be used in military and civilian applications, e.g. traffic assistance, surveillance, mapping, inspection power lines, oil pipelines, etc.

The attitude control system design of UAVs is a challenging task due to various difficulties faced when working with them. These systems are multi-input multi output (MIMO), nonlinear, coupled between the longitudinal and lateral dynamic, and very sensible to external disturbances. Moreover, parametric uncertainties characteristics may also cause more complications during the design of such attitude control systems.

A number of control approaches have been presented in the literatures. In [2] is presented, the output feedback control method to design the attitude control system for UAV. It is shown in [3] that a roll-channel fractional order proportional integral flight controller for a small fixed wing UAV is designed. The fuzzy sliding mode control based on the multi objective genetic algorithm is presented in [4] to design the altitude autopilot of UAV. The authors of [5] proposed an $\mathrm{L}_{1}$ adaptive controller as autopilot inner loop controller candidate, designed and tested its controller based on piecewise constant adaptive laws. Navigation outer loop parameters are regulated via PID control method. In [6], the attitude tracking system is designed for a small quad rotor UAV through model reference adaptive control method. The main feature of [7] is that the adaptive controller is designed, assuming that all of the nonlinear functions of the system have uncertainties, and the neural network weights are adjusted adaptively via Lyapunov theory. In [8], feedback linearization 
and model reference adaptive control (MRAC) are integrated to design the attitude control system for a fixed wing UAV.

Sliding mode control (SMC) has been suggested as a powerful approach for control systems with nonlinearities, uncertain dynamics and bounded input disturbances. The most distinguished feature of SMC is its ability to provide fast error convergence and strong robustness for control systems in the sense that the closed loop systems are completely insensitive to nonlinearities and uncertain dynamics [9].

However, the bounds of system uncertainties are required for sliding mode control and this drawback attenuates the control system performance. In this paper, due to a nonlinear dynamic and the presence of uncertainties, a sliding mode control approach based on adaptive control is investigated for nonlinear coupling dynamic of UAV. The approach does not need the upper bound of parametric uncertainty and disturbance. It also guaranties small fixed UAV attitude stabilization while offering altitude trajectory tracking. In the proposed scheme, the unknown sliding mode control parameters are approximated via adaptation laws. The stability of the control system is demonstrated based on lyapunov theory. Adaptive sliding mode control (ASMC), the combination of adaptive control method and SMC approach, is more flexible and convenient in controller design than SMC.

In comparison with other control approaches, the proposed method benefits from high robustness in presence of different parametric uncertainties, i.e. aerodynamic coefficients, inertia moment and configuration parameters uncertainties and disturbances caused by the environment such as wind. Furthermore, the chattering phenomenon in sliding mode control is avoided by using saturation function.

The paper is organized as follows: In section 2, dynamic and kinematic equations of small fixed wing UAV for dynamic modeling are introduced. The control strategy for nonlinear dynamics of UAV is investigated in section 3. In section 4, the adaptive sliding mode controller theory is presented. Simulation results are analyzed in section 5. Finally, conclusions are presented in section 6.

\section{DYNAMIC MODELING}

It is assumed in modeling that UAV is a rigid body with a symmetric geometry. The center of mass is also assumed to be fixed. Attitude of a rigid body moving in space is expressed in Euler angles (roll-pitchyaw), based on a body frame as shown in Fig. 1. The control of a fixed wing small UAV is represented by three control surfaces: aileron, elevator and rudders, and the thrust generated by an engine.

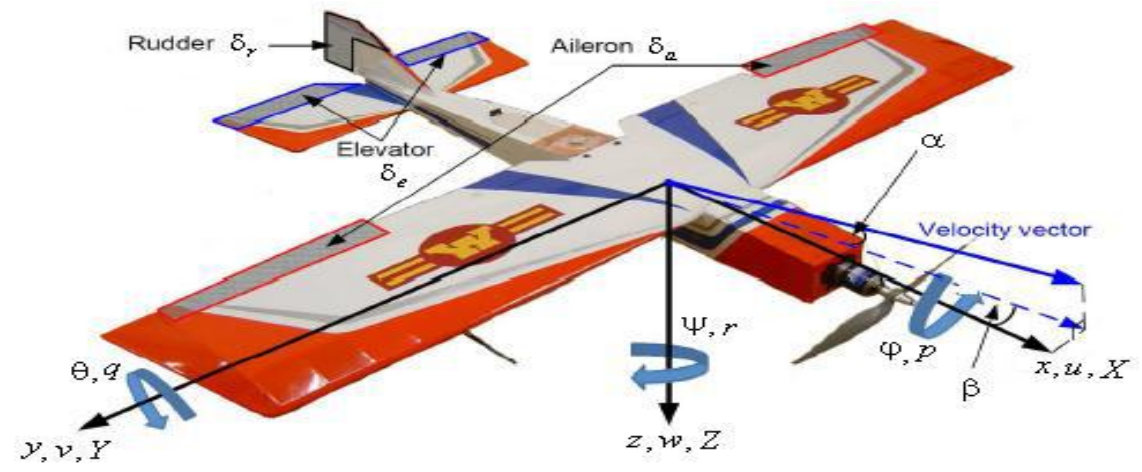

Figure 1. The UAV on body frame

The UAV 6-DOF flight dynamic equations of motion are derived from the Newton-Euler laws [10] as follow:

$$
\begin{aligned}
& m(\dot{u}-v r+w q)=X_{A}-m g \sin \theta+T \\
& m(\dot{v}+u r-w p)=Y_{A}+m g \cos \theta \sin \phi \\
& m(\dot{w}-u q+v p)=Z_{A}+m g \cos \theta \cos \phi \\
& I_{x x} \dot{p}-I_{x z} \dot{r}-I_{x z} p q+\left(I_{z z}-I_{y y}\right) r q=L_{A} \\
& I_{y y} \dot{q}+\left(I_{x x}-I_{z z}\right) p r+I_{x z}\left(p^{2}-r^{2}\right)=M_{A} \\
& I_{z z} \dot{r}-I_{x z} \dot{p}+\left(I_{y y}-I_{x x}\right) p q+I_{x z} q r=N_{A}
\end{aligned}
$$


Where $u, v, w$, are the velocity projections along the body frame, $p, q, r$ are the projections of the UAV angular rate $\underset{\omega}{\rightarrow}$ along body frame axes, $T$ is the UAV thrust force, $\phi, \theta, \psi$ are the attitude angles (roll, pitch, and yaw), $\underset{g}{\rightarrow}$ is the gravitational acceleration, $\mathrm{I}_{i j}$ represents the inertia moments, and is the UAV mass. The aerodynamic forces $\mathrm{X}_{\mathrm{A}}, \mathrm{Y}_{\mathrm{A}}, \mathrm{Z}_{\mathrm{A}}$, and moments $\mathrm{L}_{\mathrm{A}}, \mathrm{M}_{\mathrm{A}}, \mathrm{N}_{\mathrm{A}}$ can then be calculated by means of aerodynamic coefficients as:

$$
\begin{array}{lll}
L_{A}=\frac{1}{2} \rho V^{2} S b c_{l}, & M_{A}=\frac{1}{2} \rho V^{2} S \bar{c} c_{m}, & N_{A}=\frac{1}{2} \rho V^{2} S b c_{n} \\
X_{A}=\frac{1}{2} \rho V^{2} S C_{X}, & Y_{A}=\frac{1}{2} \rho V^{2} S C_{Y}, & Z_{A}=\frac{1}{2} \rho V^{2} S C_{Z}
\end{array}
$$

$V=\sqrt{u^{2}+v^{2}+w^{2}}$ Where

is the airspeed and wing surface area, $\mathrm{S}$, the wingspan, $\mathrm{b}$, the mean aerodynamic cord, $\bar{c}$ and the air density, $\rho$, are considered constant parameters. The dimensionless coefficients in the force/moment expressions can be decomposed in the following set of equations [11]:

$$
\begin{aligned}
& C_{X}=C_{L} \sin \alpha-C_{D} \cos \alpha, C_{Z}=-C_{D} \sin \alpha-C_{L} \cos \alpha, C_{Y}=C_{Y_{\beta}} \beta+C_{Y_{\delta r}} \delta r+\frac{b}{2 V_{a}}\left(C_{Y_{p}} p+C_{Y_{r}} r\right) \\
& c_{l}=c_{l_{\beta}} \beta+c_{l_{\delta a}} \delta a+c_{l_{\delta r}} \delta r+\frac{b}{2 V_{a}}\left(c_{l_{p}} p+c_{l_{r}} r\right) \\
& c_{m}=c_{m 0}+c_{m_{\alpha}} \alpha+c_{m_{\delta e}} \delta e+\frac{\bar{c}}{2 V_{a}}\left(c_{m_{q}} q+c_{m_{\dot{\alpha}}} \dot{\alpha}\right) \\
& c_{n}=c_{n_{\beta}} \beta+c_{n_{\delta a}} \delta a+c_{n_{\delta r}} \delta r+\frac{b}{2 V_{a}}\left(c_{n_{p}} p+c_{n_{r}} r\right)
\end{aligned}
$$

Where the lift $\left(C_{L}\right)$ and the drag $\left(C_{D}\right)$ coefficients are calculated using the following equations:

$$
C_{L}=C_{L 0}+C_{L_{\alpha}} \alpha+C_{L_{\delta e}} \delta e+\frac{\bar{c}}{2 V_{a}} C_{L_{q^{\prime}}} \quad C_{D}=C_{D 0}+C_{D_{\delta r}} \delta r+C_{D_{\delta e}} \delta e+\frac{C_{L}-C_{L_{\min }}}{\pi e A R}
$$

Where are $\alpha, \beta$ the attack and the sideslip angles. $\delta e, \delta a$, and $\delta r$ represent the moving surfaces of elevator, ailerons, and rudder, respectively. Moreover, $e$ is the Oswalds efficient number, and $A R$ is the aspect ratio calculated as $A R=\frac{b^{2}}{2}[11]$

The kinematics of the aircraft rotation motion relating body angular rates, Euler angles and aerodynamic angles are given by:

$$
\begin{aligned}
& \dot{\phi}=p+\tan \theta(q \sin \phi+r \cos \phi) \\
& \dot{\theta}=q \cos \phi-r \sin \phi \\
& \dot{\psi}=\frac{q \sin \phi+r \cos \phi}{\cos \theta}
\end{aligned}
$$

According to following equation, actuator dynamics are second order functions and the control surface bounds are within -20 and +20 degrees.

$$
G=\frac{\omega_{a}^{2}}{s^{2}+2 \xi_{a} \omega_{a}+\omega_{a}^{2}}
$$

Where $\omega_{\mathrm{a}}$ (the natural frequency) and $\xi_{\mathrm{a}}$ (damping ratio), are $45 \mathrm{rad} / \mathrm{s}$ and 0.7 for all control surfaces, respectively [11].

\section{CONTROL STRATEGY}

As illustrated in Fig. 2, the multivariable dynamic control system for a fixed wing UAV is divided into longitudinal and lateral plans. The longitudinal plan controls the pitch angle $(\theta)$ in the inner loop and the altitude $(\mathrm{h})$ in the outer loop by elevator control surface $\left(\delta_{\mathrm{e}}\right)$ and controls the speed by throttle $\left(\delta_{\mathrm{t}}\right)$. The lateral 
plans controls the roll angle $(\phi)$ in the inner loop and heading angle $(\psi)$ in the outer loop by aileron control surface $\left(\delta_{\mathrm{a}}\right)$. In this paper, the adaptive sliding mode controller is chosen to control the inner navigation loops $(\theta, \phi)$ those need a faster response and are more prone to be affected by parametric uncertainties. Altitude $h$ and heading $\psi$ outer navigation loops are instead controlled by simple PIDs. It should be noted that although no rudder is used, the response on the heading angles is still satisfying with aileron control.

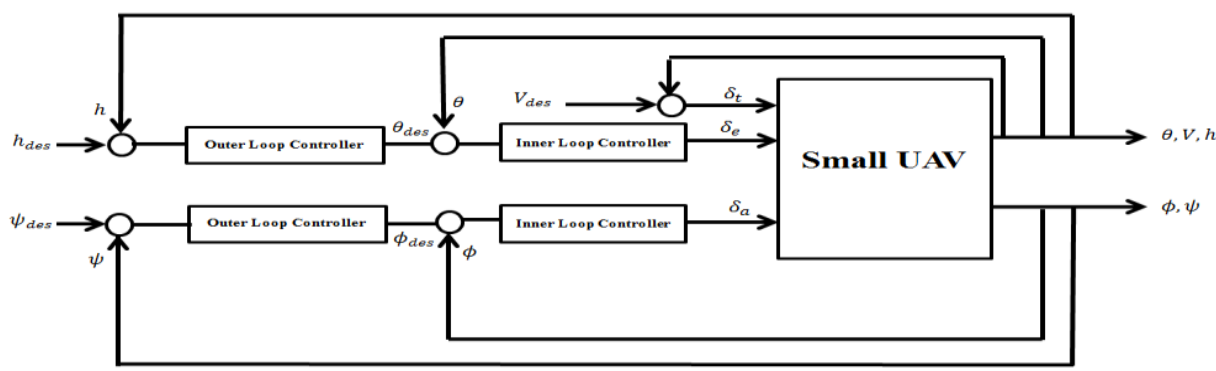

Figure 2. The control scheme

\section{ADAPTIVE SLIDING MODE CONTROL OF SMALL UAV}

This section consist of two parts. At first, the sliding mode control law is designed for the two longitudinal and lateral plans. Next, the unknown system parameters are approximated via adaptation laws by introducing new lyapunov functions.

\subsection{Sliding Mode Control of Small UAV}

In order to apply sliding mode longitudinal and lateral controllers, we can define the sliding surface as

$$
\mathrm{s}(\mathrm{t})=\mathrm{e}_{1}+\mu \mathrm{e}_{2}
$$

Where $\mathrm{p}(\mathrm{t})-\mathrm{p}_{\mathrm{d}}(\mathrm{t})$

$$
\mathrm{s}(\mathrm{t})=\left[\mathrm{s}_{1}(\mathrm{t}), \mathrm{s}_{2}(\mathrm{t})\right], \mathrm{e}_{1}=\left[\mathrm{e}_{\theta}, \mathrm{e}_{\phi}\right], \mathrm{e}_{2}=\left[\mathrm{e}_{\mathrm{q}}, \mathrm{e}_{\mathrm{p}}\right], \mu=\mathrm{e}_{\theta}=\theta(\mathrm{t})-\theta_{\mathrm{d}}(\mathrm{t}), \mathrm{e}_{\phi}=\phi(\mathrm{t})-\phi_{\mathrm{d}}(\mathrm{t}), \mathrm{e}_{\mathrm{q}}=\mathrm{q}(\mathrm{t})-\mathrm{q}_{\mathrm{d}}(\mathrm{t}), \mathrm{e}_{\mathrm{p}}=
$$

In the above expressions, is a positive design parameter and accounts for the desired amount of variables. The sliding mode can then be differentiated with respect to time as:

$$
\dot{s}(t)=\dot{e}_{1}+\mu \dot{e}_{2}
$$

As $\delta_{e}$ and $\delta_{\mathrm{a}}$ are derived from, $\dot{p}$ and $\dot{q}$, equation (2) can be reordered as following:

$$
\begin{aligned}
& \dot{q}=\Gamma_{5} p r-\Gamma_{6}\left(p^{2}-r^{2}\right)+\frac{\rho V^{2} S c}{2 I_{y}}\left(C_{m 0}+C_{m \alpha} \alpha+C_{m q} \frac{c q}{2 V_{a}}+C_{m \delta_{e}} \delta_{e}\right) \\
& \dot{p}=\Gamma_{1} p q-\Gamma_{2} q r+\frac{1}{2} \rho V^{2} S b\left(C_{p_{\beta}} \beta+C_{p_{p}} \frac{b p}{2 V}+C_{p_{r}} \frac{b r}{2 V}+C_{p_{\delta_{a}}} \delta_{a}\right)
\end{aligned}
$$

Where

$$
\begin{aligned}
& \Gamma=I_{x x} I_{z z}-I_{x z}^{2}, \Gamma_{1}=\frac{I_{x z}\left(I_{x x}-I_{y y}+I_{z z}\right)}{\Gamma}, \Gamma_{2}=\frac{I_{z z}\left(I_{z z}-I_{y y}\right)+I_{x z}{ }^{2}}{\Gamma}, \Gamma_{3}=\frac{I_{z z}}{\Gamma}, \Gamma_{4}=\frac{I_{x z}}{\Gamma}, \\
& \Gamma_{5}=\frac{I_{z z}-I_{x x}}{\Gamma}, \Gamma_{6}=\frac{I_{x z}}{I_{y y}} \\
& \mathrm{C}_{\mathrm{p}_{\beta}}=\Gamma_{3} \mathrm{C}_{\mathrm{l}_{\beta}}+\Gamma_{4} \mathrm{C}_{\mathrm{n}_{\beta}}, \mathrm{C}_{\mathrm{p}_{\mathrm{p}}}=\Gamma_{3} \mathrm{C}_{\mathrm{l}_{\mathrm{p}}}+\Gamma_{4} \mathrm{C}_{\mathrm{n}_{\mathrm{p}}}, \mathrm{C}_{\mathrm{p}_{\mathrm{r}}}=\Gamma_{3} \mathrm{C}_{\mathrm{l}_{\mathrm{r}}}+\Gamma_{4} \mathrm{C}_{\mathrm{n}_{\mathrm{r}}}, \mathrm{C}_{\mathrm{p}_{\delta_{\mathrm{a}}}}=\Gamma_{3} \mathrm{C}_{\mathrm{l}_{\delta_{\mathrm{a}}}}+\Gamma_{4} \mathrm{C}_{\mathrm{n}_{\delta_{\mathrm{a}}}}
\end{aligned}
$$

Now, consider a sliding mode controller described as: 


$$
\begin{aligned}
& u(1)=\Upsilon \mathrm{A}^{\mathrm{T}}+k_{1} f_{1} \\
& u(2)=\mathrm{OB}^{\mathrm{T}}+k_{1} f_{1}
\end{aligned}
$$

$$
\begin{aligned}
u & =\left[\begin{array}{llllll}
\delta_{e}, \delta_{a}
\end{array}\right] \\
r & =\left[\begin{array}{llllll}
\frac{-2 I_{y}}{\rho S c C_{m \delta_{e}}} & \frac{-2 I_{y} \Gamma_{5}}{\rho S c C_{m \delta_{e}}} & \frac{-2 I_{y} \Gamma_{6}}{\rho S c C_{m \delta_{e}}} & \frac{C_{m \mathrm{o}}}{C_{m \delta_{e}}} & \frac{C_{m \alpha}}{C_{m \delta_{e}}} & \frac{c C_{m q}}{2 C_{m \delta_{e}}}
\end{array}\right] \\
\Theta & =\left[\begin{array}{llllll}
\frac{-2}{\rho S b C_{p_{\delta_{a}}}} & \frac{-2 \Gamma_{1}}{\rho S b C_{p_{\delta_{a}}}} & \frac{-2 \Gamma_{2}}{\rho S b C_{p_{\delta_{a}}}} & \frac{-C_{p_{\beta}}}{C_{p_{\delta_{a}}}} & \frac{-b C_{p_{p}}}{2 C_{p_{\delta_{a}}}} & \frac{b C_{p_{r}}}{2 C_{p_{\delta_{a}}}}
\end{array}\right] \\
A & =\left[\begin{array}{llllll}
\frac{\dot{e}_{\theta}}{\mu_{1} V^{2}} & \frac{p r}{V^{2}} & -\frac{\left(p^{2}-r^{2}\right)}{V^{2}} & -1 & -\alpha & -\frac{q}{V}
\end{array}\right] \\
B & =\left[\begin{array}{llllll}
\frac{\dot{e}_{\phi}}{\mu_{2} V^{2}} & \frac{p q}{V^{2}} & -\frac{q r}{V^{2}} & -\beta & -\frac{p}{V} & -\frac{r}{V}
\end{array}\right]
\end{aligned}
$$

In which, $f_{i}=\operatorname{sgn}\left(s_{i}\right)$ is a sign function, and $k_{i}$ is positive constant. In order to use adaptation laws, $\Upsilon$ and $\odot$ vectors need to be assumed as unknown constants. The stability of the sliding mode control laws will be reviewed in the following.

Theorem 1. Consider the rotational dynamic of a small UAV in equations (9) and (10) with sliding surfaces given by equation (8). If the control laws of (11) and (12) are implemented, the closed-loop system will be globally and asymptotically stable, and the tracking error of attitude UAV converge to zero, i.e.

$$
\lim _{t \rightarrow \infty} e_{\theta}=0, \lim _{t \rightarrow \infty} e_{\phi}=0, \lim _{t \rightarrow \infty} e_{q}=0, \lim _{t \rightarrow \infty} e_{p}=0
$$

Proof. A candidate Lyapunov positive definite function $L(\mathrm{~s}(\mathrm{t}))$ is defined as:

$$
\begin{aligned}
& \mathcal{L}(s(t))=\frac{1}{2} s_{1}(t)^{2}+\frac{1}{2} s_{2}(t)^{2} \\
& \text { Then, if } \dot{\mathcal{L}}(\mathrm{s}(\mathrm{t})) \leq 0, \text { asymptotic Lyapunov stability will be guaranteed. In view of }(8), \text { calculating the } \\
& \text { derivative of } \mathcal{L}(\mathrm{s}(\mathrm{t})) \text { yields: } \\
& \begin{aligned}
\dot{\mathcal{L}}(s(t))= & s_{1}(t) \dot{S}_{1}(t)+s_{2}(t) \dot{s}_{2}(t)=s_{1}(t)\left(\dot{e}_{\theta}+\mu_{1} \dot{e}_{q}\right)+s_{2}(t)\left(\dot{e}_{\phi}+\mu_{2} \dot{e}_{p}\right) \\
& =s_{1}(t)\left(\dot{e}_{\theta}+\mu_{1} \dot{e}_{q}\right)+s_{2}(t)\left(\dot{e}_{\phi}+\mu_{2} \dot{e}_{p}\right) \\
& =s_{1}(t)\left(\dot{e}_{\theta}+\mu_{1}\left(\Gamma_{5} p r-\Gamma_{6}\left(p^{2}-r^{2}\right)+\frac{\rho V^{2} S c}{2 I_{y}}\left(C_{m o}+C_{m \alpha} \alpha+C_{m q} \frac{c q}{2 V_{a}}+C_{m \delta_{e}} \delta_{e}\right)\right)\right) \\
& +s_{2}(t)\left(\dot{e}_{\phi}+\mu_{2}\left(\Gamma_{1} p q-\Gamma_{2} q r+\frac{\rho V^{2} S b}{2}\left(C_{p_{\beta}} \beta+C_{p_{p}} \frac{b p}{2 V}+C_{p_{r}} \frac{b r}{2 V}+C_{p_{\delta_{a}}} \delta_{a}\right)\right)\right)
\end{aligned}
\end{aligned}
$$

Substituting the new parameters $\Upsilon$ and $\odot$ into the above equations yields:

$$
\begin{aligned}
& \dot{L}(s(t))=s_{1}(t)\left(\dot{e}_{\theta}+\frac{V^{2} \mu_{1}}{r_{1}}\left(r_{2} \frac{p r}{V^{2}}-\gamma_{3} \frac{p^{2}-r^{2}}{V^{2}}-r_{4}-r_{5} \alpha-\Upsilon_{6} \frac{q}{V_{a}}-\delta_{e}\right)\right) \\
& +s_{2}(t)\left(\dot{e}_{\phi}+\frac{V^{2} \mu_{2}}{\Theta_{1}}\left(\Theta_{2} \frac{p q}{V^{2}}-\Theta_{3} \frac{q r}{V^{2}}-\Theta_{4} \beta-\Theta_{5} \frac{p}{V}+\Theta_{6} \frac{r}{V}-\delta_{a}\right)\right)
\end{aligned}
$$

Substituting the control laws (11) and (12), we obtain:

$$
\begin{aligned}
& \dot{L}(s(t))=s_{1}(t)\left(\dot{e}_{\theta}+\frac{V^{2} \mu_{1}}{r_{1}}\left(r_{2} \frac{p r}{V^{2}}-r_{3} \frac{p^{2}-r^{2}}{V^{2}}-r_{4}-r_{5} \alpha-r_{6} \frac{q}{V_{a}}-r_{1} \frac{\dot{e}_{\theta}}{\mu_{1} V^{2}}-r_{2} \frac{p r}{V^{2}}+r_{3} \frac{p^{2}-r^{2}}{V^{2}}+r_{4}+r_{5} \alpha+r_{6} \frac{q}{V}-k_{1} f_{1}\right)\right) \\
& +s_{2}(t)\left(\dot{e}_{\phi}+\frac{V^{2} \mu_{2}}{\Theta_{1}}\left(\Theta_{2} \frac{p q}{V^{2}}-\Theta_{3} \frac{q r}{V^{2}}-\Theta_{4} \beta-\Theta_{5} \frac{p}{V}+\Theta_{6} \frac{r}{V}-\Theta_{1} \frac{\dot{e}_{\phi}}{\mu_{2} V^{2}}-\Theta_{2} \frac{p q}{V^{2}}+\Theta_{3} \frac{q r}{V^{2}}+\Theta_{4} \beta+\Theta_{5} \frac{p}{V}-\Theta_{6} \frac{r}{V}+k_{2} f_{2}\right)\right.
\end{aligned}
$$

After simplifying the above equations, we have:

$$
\dot{\mathcal{L}}(s(t)) \leq-k_{1}\left|s_{1}(t)\right|-k_{2}\left|s_{2}(t)\right|
$$

Obviously, $\dot{\mathcal{L}}(s(t)) \leq 0$. Hence, based on the Lyapunov stability theory, the theorem is proved.

\subsection{Adaptive Sliding Mode Control of small UAV}

In this section, the sliding mode control laws that were designed in the previous section are improved by means of adaptive laws. Consider adaptive sliding mode control laws () as: 


$$
\begin{aligned}
& u(1)=\Upsilon \mathrm{A}^{\mathrm{T}}+k_{1} f_{1} \\
& u(2)=\mathrm{OB}^{\mathrm{T}}+k_{1} f_{1}
\end{aligned}
$$

Where the estimated vectors^and^are updated using the following adaptation laws:

$$
\begin{aligned}
& \dot{\tilde{Y}}_{i}=\eta s_{1}(t) \frac{\mu_{1} V^{2}}{\Upsilon_{1}} A_{i} \quad i=1: 6 \\
& \dot{\tilde{\Theta}}_{j}=\vartheta s_{2}(t) \frac{\mu_{2} V^{2}}{\Theta_{1}} B_{j} \quad j=1: 6
\end{aligned}
$$

Where $\eta$ and $\vartheta$ are positive constants and the $\widetilde{\Theta}$ and $\widetilde{Y}$ vectors denote the difference between the actual and estimated vectors:

$$
\widetilde{\Theta}=\widehat{\Theta}-\Theta \Rightarrow \dot{\widetilde{\Theta}}=\dot{\hat{\Theta}}, \quad \widetilde{\Upsilon}=\widehat{\Upsilon}-\Upsilon \Rightarrow \dot{\widetilde{\Upsilon}}=\dot{\widehat{\Upsilon}}
$$

Theorem 2. Consider rotational dynamic of a small UAV described by equations (9) and (10) with unknown parameters. If control laws are designed as equations (13) and (14) with the adaptation laws (15) and (16), the trajectory of the system will track the desired trajectory and the system is globally asymptotically stable in finite time under the presence of uncertainties and disturbances.

Proof. To prove the robustness and stability of the proposed controller and to derive the estimation laws for the unknown parameters, the following Lyapunov function is considered:

$$
\begin{aligned}
& \mathcal{L}_{1}^{\prime}\left(s_{1}(t), \widetilde{r}\right)=\frac{1}{2} s_{1}(t)^{2}+\frac{1}{2 \eta} \sum_{i=1}^{6} \tilde{r}_{i}^{2}, \quad \mathcal{L}_{2}^{\prime}\left(s_{2}(t), \widetilde{\Theta}\right)=\frac{1}{2} s_{2}(t)^{2}+\frac{1}{2 \vartheta} \sum_{j=1}^{6} \widetilde{\Theta}_{j}^{2} \\
& \text { Calculating the derivative of } \mathcal{L}_{1}^{\prime} \text { along the system (8) yields: } \\
& \dot{\hat{L}}_{1}^{\prime}=s_{1}(t) \dot{s}_{1}(t)+\frac{1}{\eta} \sum_{i=1}^{6} \tilde{Y}_{i} \dot{\tilde{Y}}_{i}=s_{1}(t)\left(\dot{e}_{\theta}+\mu_{1} \dot{e}_{q}\right)+\frac{1}{\eta} \sum_{i=1}^{6} \tilde{r}_{i} \dot{\widetilde{Y}}_{i} \\
& =s_{1}(t)\left(\dot{e}_{\theta}+\frac{\mu_{1} V^{2}}{\gamma_{1}}\left(r_{2} \frac{p r}{V^{2}}-r_{3} \frac{p^{2}-r^{2}}{V^{2}}-r_{4}-\gamma_{5} \alpha-\gamma_{6} \frac{q}{V_{a}}-\delta_{e}\right)\right)+\frac{1}{\eta} \sum_{i=1}^{6} \tilde{r}_{i} \dot{\Upsilon}_{i} \\
& =s_{1}(t) \frac{\mu_{1} V^{2}}{\gamma_{1}}\left(r_{1}\left(\frac{\dot{e}_{\theta}}{\mu_{1} V^{2}}\right)+r_{2} \frac{p r}{V^{2}}-r_{3} \frac{p^{2}-r^{2}}{V^{2}}-\gamma_{4}-\gamma_{5} \alpha-\Upsilon_{6} \frac{q}{V_{a}}-\hat{\Upsilon}_{1}\left(\frac{\dot{e}_{\theta}}{\mu_{1} V^{2}}\right)-\widehat{\Upsilon}_{2} \frac{p r}{V^{2}}+\hat{\Upsilon}_{3} \frac{\left(p^{2}-r^{2}\right)}{V^{2}}\right. \\
& \left.\left.+\hat{\Upsilon}_{4}+\hat{\Upsilon}_{5} \alpha+\hat{\Upsilon}_{6} \frac{q}{V_{a}}-k_{1} f_{1}\right)\right)+\frac{1}{\eta} \sum_{i=1}^{6} \tilde{r}_{i} \dot{\Upsilon}_{i} \\
& =s_{1}(t) \frac{\mu_{1} V^{2}}{r_{1}}\left(-\tilde{r}_{1}\left(\frac{\dot{e}_{\theta}}{\mu_{1} V^{2}}\right)-\tilde{\Upsilon}_{2} \frac{p r}{V^{2}}+\tilde{Y}_{3} \frac{p^{2}-r^{2}}{V^{2}}+\tilde{Y}_{4}+\tilde{r}_{5} \alpha+\tilde{\Upsilon}_{6} \frac{q}{V}-k_{1} \operatorname{sgn}\left(s_{1}\right)\right)+\frac{1}{\eta} \sum_{i=1}^{6} \tilde{r}_{i} \dot{\Upsilon}_{i}
\end{aligned}
$$

Substituting the adaptation laws (15) into the above equation yields:

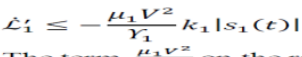

$$
\begin{aligned}
& \text { The term } \frac{\mu_{1} v z}{\gamma_{2}} \text { on the right hand side of this equation is positive. Hence: } \hat{H}_{i}\left(S_{1}(t), \tilde{Y}\right) \leq 0 \text {, and on account of } \\
& \text { the Lyapunov stability theory, the theorem is proved. } \\
& \hat{L}_{2}=s_{2}(t) s_{2}(t)+\frac{1}{V} \sum_{j=1}^{0} \hat{\theta}_{j} \dot{\theta}_{j}=s_{2}(t)\left(\dot{e}_{\phi}+\mu_{2} \dot{e}_{p}\right)+\frac{1}{V} \sum_{j=1}^{0} \hat{\theta}_{j} \dot{\theta}_{j} \\
& =s_{2}(t)\left(e_{\phi}+\frac{V^{2} \mu_{2}}{\Theta_{1}}\left(\Theta_{2} \frac{p q}{V^{2}}-\Theta_{3} \frac{q r}{V^{2}}-\Theta_{4} \beta-\Theta_{5} \frac{p}{V}+\theta_{0} \frac{r}{V}-\delta_{a}\right)\right)+\frac{1}{v} \sum_{j=1}^{0} \theta_{1} \dot{\theta}_{j} \\
& =s_{2}(t) \frac{\mu_{2} V^{2}}{\Theta_{1}}\left(\Theta_{1}\left(\frac{\dot{e}_{\phi}}{\mu_{2} V^{2}}\right)+\Theta_{2} \frac{p q}{V^{2}}-\Theta_{3} \frac{q r}{V^{2}}-\Theta_{4} \beta-\Theta_{5} \frac{p}{V}+\Theta_{0} \frac{r}{V}-\Theta_{1}\left(\frac{\dot{e}_{\phi}}{\mu_{2} V^{2}}\right)-\Theta_{2} \frac{p q}{V^{2}}+\Theta_{3} \frac{q r}{V^{2}}+\Theta_{4} \beta\right. \\
& \left.+\theta_{5} \frac{p}{V}-\theta_{0} \frac{r}{V}-k_{2} f_{2}\right)+\frac{1}{\mathfrak{V}} \sum_{j=1}^{\sigma} \sigma_{1} \dot{\theta}_{j}
\end{aligned}
$$

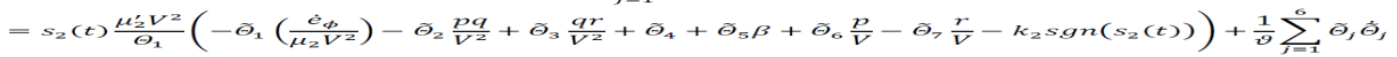

With substituting the adaptation laws (16) yields

$$
\begin{aligned}
& \dot{\mathcal{L}}_{2}^{\prime} \leq-\frac{\mu_{2}^{\prime} V^{2}}{\Theta_{1}} k_{2}\left|s_{2}(t)\right| \\
& \text { Similarly, as } \frac{\mu_{2}^{\prime} V^{2}}{\theta_{1}} \text { is positive, } \dot{\mathcal{L}}_{2}^{\prime}\left(s_{2}(t), \tilde{\Theta}\right) \leq 0 \text {. Therefore, based on the Lyapunov stability theory, the } \\
& \text { theorem is proved. }
\end{aligned}
$$


Remark 1. In order to avoid the chattering phenomenon due to the imperfect implementation of the sign function in the control laws (13) and (14),the following saturation function is introduced:

In which $\varepsilon$ is a small constant.

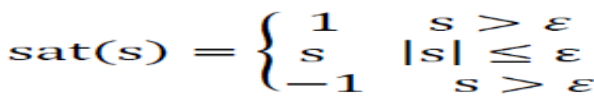

\section{SIMULATION RESULT AND DISCUSSIONS}

In this section, we present results of applying the proposed control scheme to a full 6 degree of freedom model of UAV. A mathematical model of a small fixed wing UAV has been derived from [11] and implemented in Matlab Simulink enviroment. A summary of the UAV platform physical properties is given in Table 1.

Table 1. Small fixed wing UAV parameters

\begin{tabular}{ccc}
\hline Parameter & Value & Unit \\
\hline Weight & 1.595 & $\mathrm{~kg}$ \\
Span & 1.27 & $\mathrm{~m}$ \\
Wing surface & 0.3097 & $\mathrm{~m}^{2}$ \\
Mean aerodynamic chord & 0.25 & $\mathrm{~m}$ \\
Inertia moment $I_{x x}$ & 0.0894 & $\mathrm{~kg} . \mathrm{m}^{2}$ \\
Inertia moment $I_{y y}$ & 0.1444 & $\mathrm{~kg} . \mathrm{m}^{2}$ \\
Inertia moment $I_{z z}$ & 0.1620 & $\mathrm{~kg} . \mathrm{m}^{2}$ \\
Inertia moment $I_{x z}$ & 0.014 & $\mathrm{~kg} . \mathrm{m}^{2}$ \\
\hline
\end{tabular}

The initial conditions of the state variables are $h(0)=100 \mathrm{~m}, \theta(0)=\phi(0)=\psi(0)=0 \mathrm{deg}, p(0)=q(0)=$ $\mathrm{r}(0)=0 \mathrm{deg} / \mathrm{s}, V(0)=17 \mathrm{~m} / \mathrm{s}$. In addition, the initial values applied in adaptive laws are $\widehat{3}(0)=0.7^{*} \Upsilon$, $\widehat{\odot}(0)=0.7 * \widehat{\odot}$.

In order to demonstrate the performance of the ASMC algorithm, another configuration based on the PID controllers, for both the inner and outer loops, is used in the nonlinear simulation model. PIDs gains are tuned by trial and error.

As a means to clearly demonstrate the actual responses of the system variables and the tracking trajectory, we first simulate the situation without the disturbances. Results of the ASMC and the PID algorithm for the inner loops control of small fixed wing UAV are depicted in Fig.3.

The ASMC controller outputs show an excellent tracking of the reference signals for the pitch and roll angles in comparison with the PID controller. Coupling effect of longitudinal and lateral plans results in an overshoot in the PID controller, while the proposed method in this article is free of these drawbacks. In addition, Fig. 4 shows that elevator and aileron deflections both algorithms remain under the imposed command saturation limit of 20 degrees. It is illustrated clearly in Figs. 5 and 6 that the designed adaption laws using Lyapunov method had been able to estimate unknown parameters, according to initial values of parameters and gain of adaptation laws, which are defined by the operator.

The comparison of the results of the inner loops system with the ASMC and the PID algorithms in the presence of $30 \%$ and $20 \%$ uncertainties in the aerodynamic coefficients and the Inertia moment, respectively, are illustrated in figure 6 . In addition, the disturbances are represented by wind external currents in $\mathrm{x}, \mathrm{y}$ directions with a magnitude of $5 \mathrm{~m} / \mathrm{s}$ at $\mathrm{t}=8 \mathrm{~s}$. The control inputs are shown in figure 8 . It can be concluded that the ASMC controller provide a more robust closed loop system against the uncertainties and disturbances.

Figure 13 illustrates the full nonlinear dynamic responses of the UAV for the applied hybrid ASMC algorithm in comparison with the PID controller. According to this figure, both controllers are able to track the desired trajectories. As far as the stability of the angular rates, the elevator and aileron control surfaces for both controllers in figure 14 confirm this fact. However, the PID controller shows a poor performance on reference changes due to the coupling effect of longitudinal and lateral plans, while the ASMC hybrid model acts better with a more robust inner loop.

To verify the proposed method, the UAV is tested in the presence of $20 \%$ uncertainties and wind disturbances with a magnitude of $5 \mathrm{~m} / \mathrm{s}$ at $\mathrm{t}=8 \mathrm{~s}$ in $\mathrm{x}$ direction. As it can be seen, the adaptive sliding mode control acting on the UAV results an increase in the robustness of the system against uncertainties and disturbances, while the poor performance of the PID linear controller caused the UAV to diverge from its 
path. The stability of the angular rates, the elevator and aileron control surfaces, presented in fig.15 and 16 for the ASMC method, compared to the PID method, confirm the obtained results.
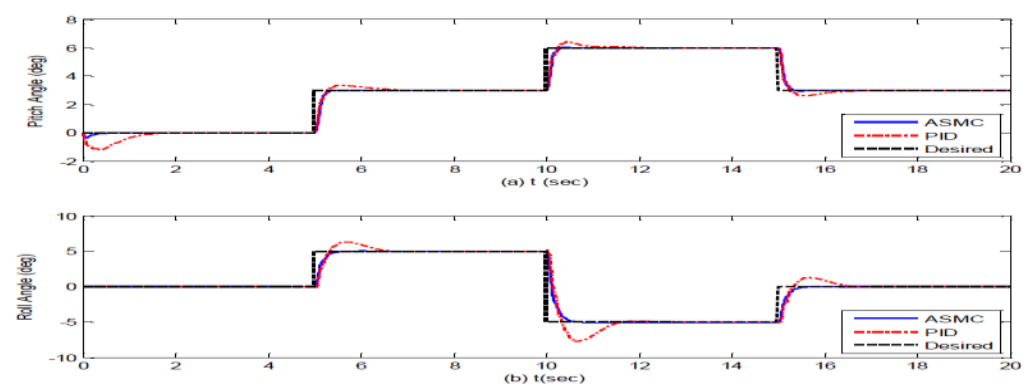

Figure 3. Inner loops variables, PID and ASMC confrontation
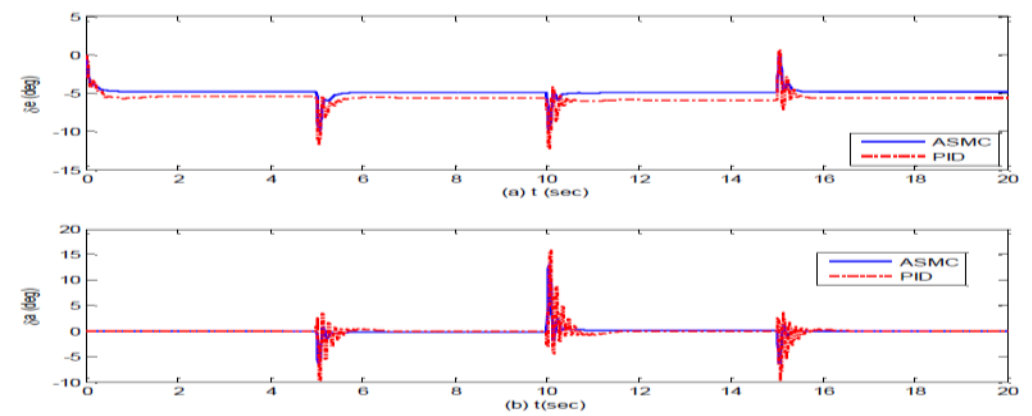

Figure 4. Inner loops control surfaces, PID and ASMC confrontation
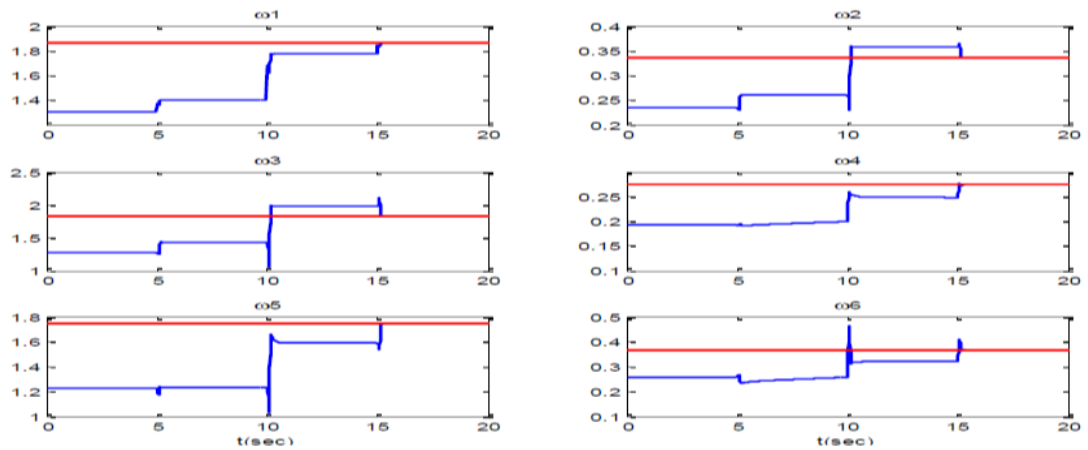

Figure 5. Parameters estimation of lateral plane 

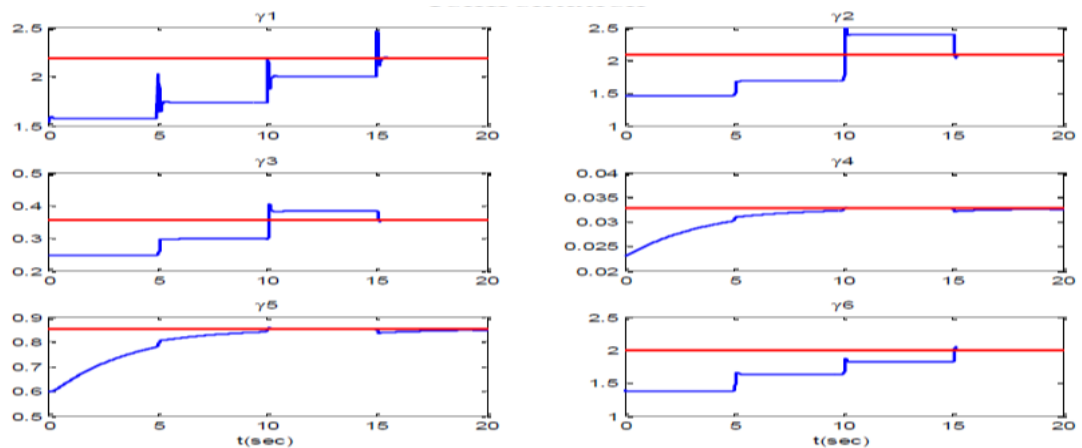

Figure 6. Parameters estimation of longitudinal plane
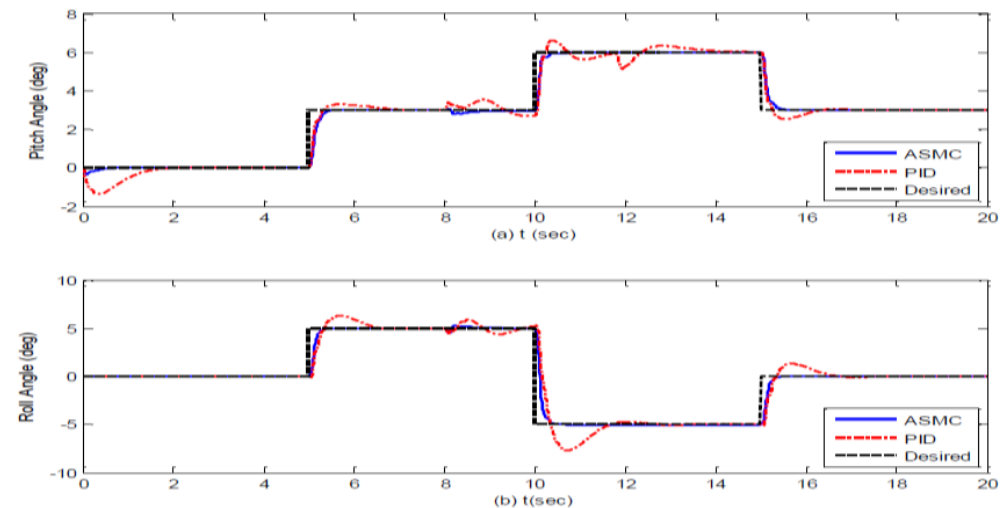

Figure 7. Inner loops variables under uncertainties and disturbance, PID and ASMC algorithms confrontation
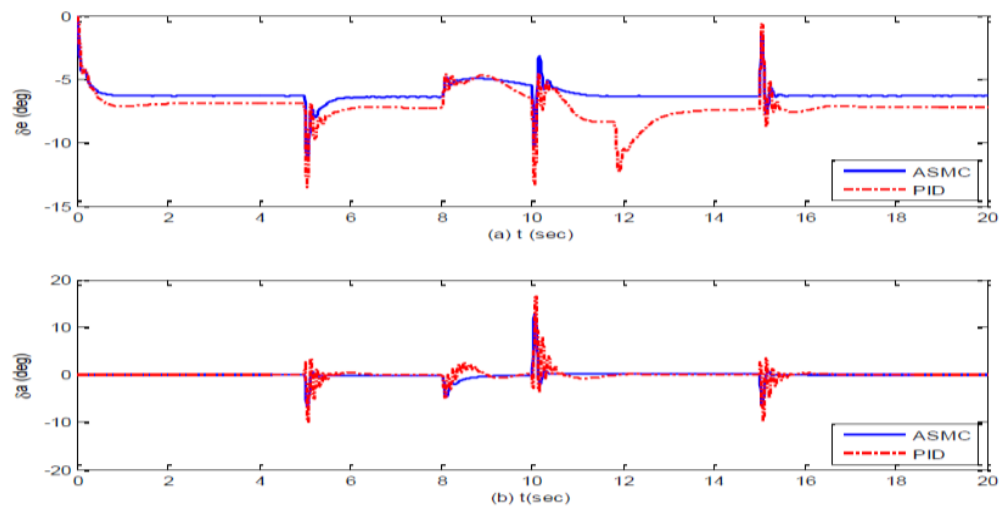

Figure 8. Inner loops control surfaces under uncertainties and disturbance, PID and ASMC algorithms confrontation
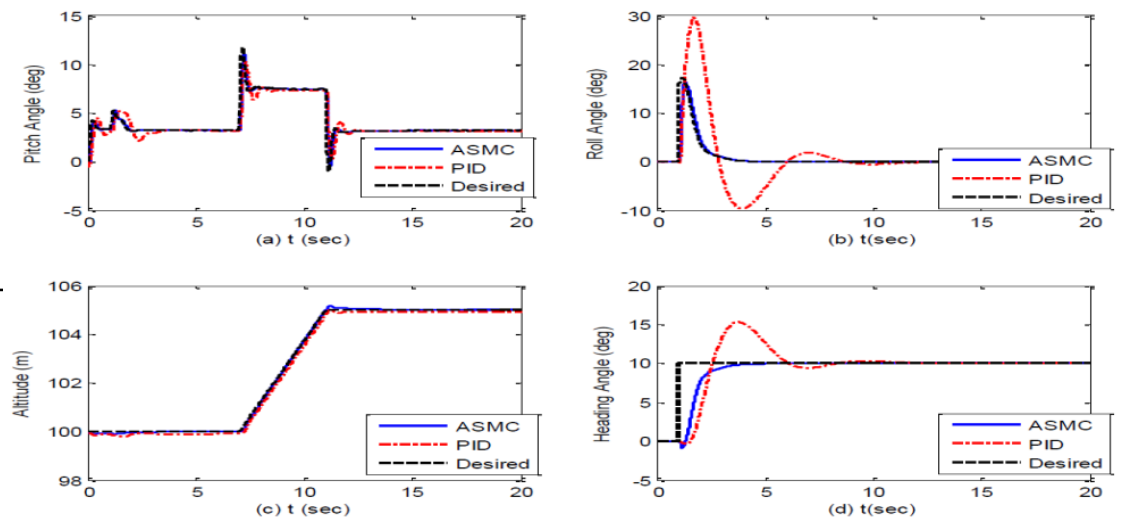

(S. Amini) 
Figure 9. Nonlinear model variables, PID and ASMC algorithms confrontation
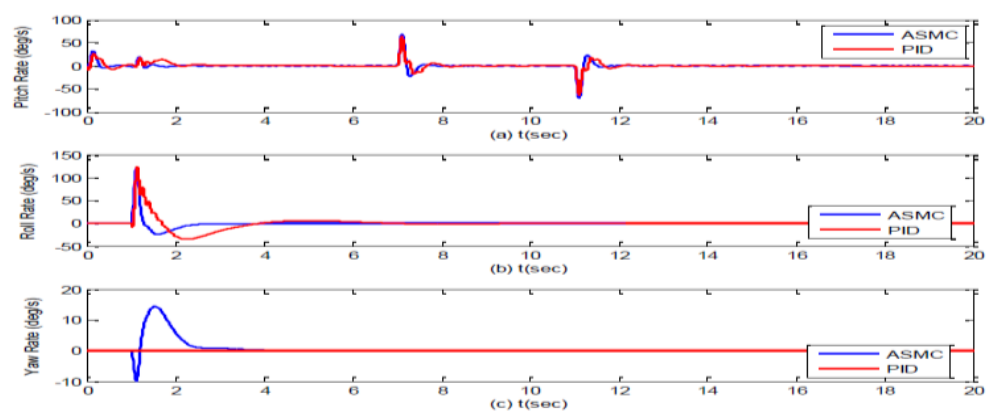

Figure 10. Nonlinear model angular rates, PID and ASMC algorithms confrontation
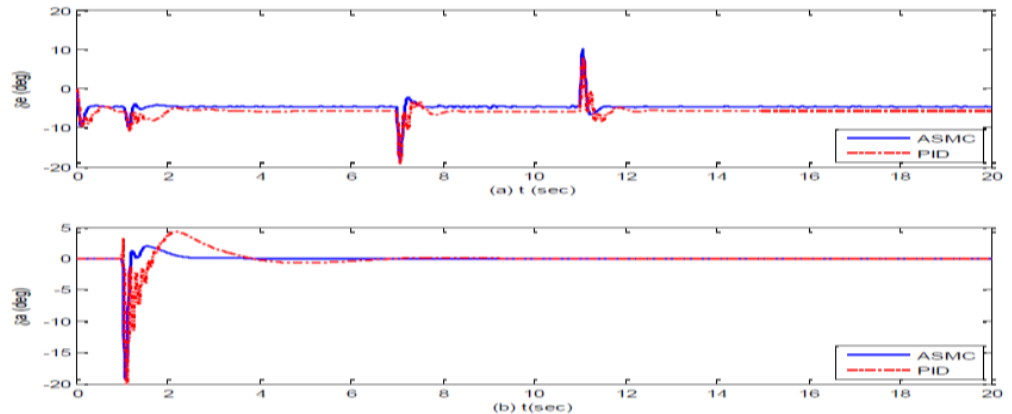

Figure 11. Nonlinear model control surfaces, PID and ASMC algorithms confrontation
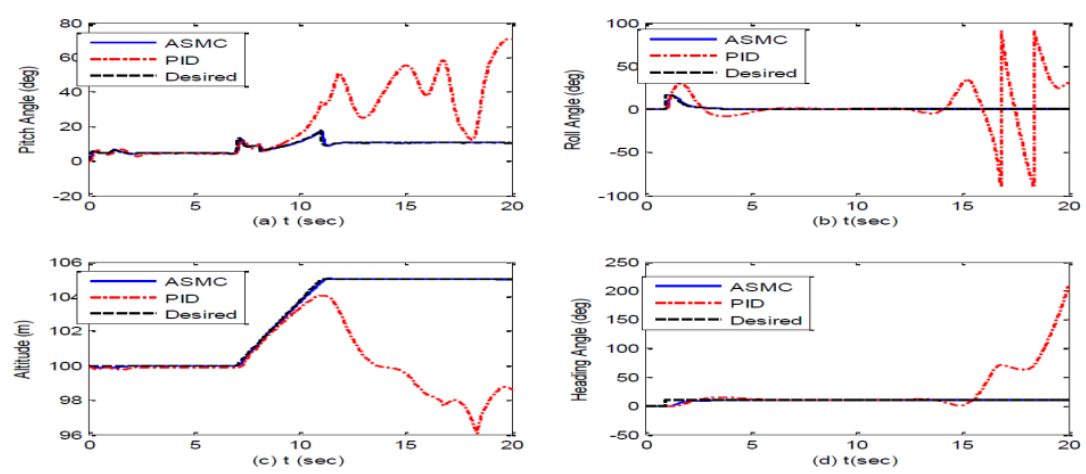
Figure 12. Nonlinear model variables under uncertainties and disturbance, PID and ASMC algorithms confrontation

\section{CONCLUSIONS}

In this paper, an adaptive sliding mode algorithm used as inner loops controller for longitudinal and lateral plans of small fixed wing UAV is analyzed. Firstly, the mathematical model of UAV's attitude motion is derived from the Newton-Euler formulation, including the kinematics and dynamics equations. The modeling of UAV is implemented in Matlab Simulink environment. Then, sliding mode control laws are designed and the unknown parameters of the corresponding controller are estimated through adaptive laws to achieve the control objective. The global asymptotical stability of the closed-loop system is proved by a Lyapunov based stability analysis. PID control laws are applied in model for altitude and heading outer loop tracking. The simulation results indicate that the attitude and altitude control system can achieve excellent tracking of the reference signals and strong robustness with respect to parametric uncertainties and external disturbance.

\section{REFERENCES}

[1] Clough, B. T. "Unmanned aerial vehicles: autonomous control challenges, a researcher's perspective." Journal of Aerospace Computing, Information, and Communication 2, vol. 8, pp. 327-347, 2005

[2] M. Lorenzo, N. Roberto, and I. Alberto, "High-gain output feedback for a miniature UAV", Journal of Robust and Nonlinear Control, no. 6, vol. 24, pp. 1104-1126, 2013.

[3] H. Chao,Y. Luo, L.Di, andY.Q.Chen, "Roll-channel fractional order controller design for a small fixed-wing unmanned aerialvehicle", Control Engineering Practice, vol. 18, no. 7, pp. 761-772,2010.

[4] A. R. Babaei, M. Mortazavi, and M. H. Moradi, "Classical and fuzzy-genetic autopilot design for unmanned aerial vehicles", Applied Soft Computing Journal, vol. 11, no. 1, pp. 365-372, 2011.

[5] E. Capello, G. Guglieri, F. Quagliotti, and D. Sartori, "Design and validation of an L1 adaptive controller for miniUAVautopilot", Journal of Intelligent and Robotic Systems, vol. 69, pp.109-118, 2013.

[6] B. T.WhiteheadandS. R. Bieniawski, "Model reference adaptivecontrol of a quadrotor UAV", in Proceedings of the AIAA Guidance,Navigation, and Control Conference, Toronto, Canada,2010.

[7] Shin, D.H., Kim, Y. ,"Reconfigurable flight control system design using adaptive neural networks", IEEETrans. Control Syst. Technol, no. 12, vol. 1,pp. 87-100,2004.

[8] Zhou, Wenya, et al. "Design of Attitude Control System for UAV Based on Feedback Linearization and Adaptive Control." Mathematical Problems in Engineering, 2014.

[9] Alessandro P, Elio U. Sliding mode control: a survey with applications in math. Journal of Mathematics and Computers in Simulation, no. 81, pp. 954-979, 2011.

[10] Roskam, "Airplane flight dynamics and automatic flights", Pt. 1, DARcorporation, 1995.

[11] Paw Y. C, "Synthesis and Validation of Flight Control for UAV", Dissertation submitted to the Faculty of the Graduate School of the University of Minnesota, December 2009. 